\title{
Effects of human umbilical cord mesenchymal stem cells derived from exosomes on migration ability of endometrial glandular epithelial cells
}

\author{
YING FENG, FULIANG ZHAN, YANYING ZHONG and BUZHEN TAN \\ Department of Gynaecology and Obstetrics, The Second Affiliated Hospital of Nanchang University, \\ Nanchang, Jiangxi 330006, P.R. China
}

Received September 7, 2019; Accepted April 6, 2020

DOI: $10.3892 / \mathrm{mmr} .2020 .11137$

\begin{abstract}
The present study aimed to investigate the effects of human umbilical cord mesenchymal stem cells (Huc-MSCs)-derived exosomes on the migratory abilities of endometrial glandular epithelial cells, and to evaluate the underlying mechanism from the perspective of epithelial-mesenchymal transition (EMT). Huc-MSCs were prepared from human umbilical cord, and eutopic endometrial glandular epithelial cells were isolated from patients with endometriosis. The exosomes derived from Huc-MSCs (Huc-MSCs-exo) were prepared using an exosome extraction kit. The endometrial glandular epithelial cells were randomly divided into two groups: Huc-MSCs-exo and control. Cell migratory ability was assessed and western blotting was used to detect the expression levels of EMT. The results of the present study demonstrated that Huc-MSCs-exo treatment significantly enhanced the migration of endometrial glandular epithelial cells from patients with endometriosis $(\mathrm{P}<0.05)$. The present study also demonstrated that treatment with Huc-MSCs-exo inhibited the expression levels of E-cadherin and promoted the expression levels of Vimentin and N-cadherin at both the mRNA and protein level. The results of the current study indicate that Huc-MSCs-exo enhance the migratory ability of endometrial glandular epithelial cells via promotion of EMT.
\end{abstract}

\section{Introduction}

Although endometriosis is considered to be a benign disease, its characteristics (including ectopic invasion and high recurrence) endow it with the biological behavior of malignant tumors $(1,2)$, of which ovaries and uterosacral ligaments are

Correspondence to: Dr Buzhen Tan, Department of Gynaecology and Obstetrics, The Second Affiliated Hospital of Nanchang University, 1 Mingde Road, Nanchang, Jiangxi 330006, P.R. China E-mail: tanbuzhen1590@163.com

Key words: human umbilical cord mesenchymal stem cells, exosomes, endometrial glandular epithelial cells, epithelialmesenchymal transition the most common locations. Symptoms of endometriosis include chronic pelvic pain, infertility and adnexal cystic mass $(3,4)$. Adhesion-invasion-implantation is the pathophysiological process underlying endometriosis and involves early cell detachment from the primary lesion and dissemination. At the later stages, circulating cells anchor and proliferate in distant tissues to form heterotopic lesions (5).

Mesenchymal stem cells (MSCs) are located in a number of tissues, such as adipose tissue and bone marrow (6). MSCs can migrate from the original tissue to the site of pathophysiological changes in response to inducing factors, such as intercellular adhesion molecule-1 (7). Human umbilical cord MSCs (Huc-MSCs) are active throughout pregnancy. Exosomes are membrane bound extracellular vesicles that are produced in the endosomal compartment of eukaryotic cells, they serve as important biomarkers to identify different diseases and as therapeutic targets for diseases (8). Exosomes derived from MSCs contain abundant biological information, including proteins, such as membrane receptors, ribosomes and genetic information. These regulate tissue regeneration and repair, immunoregulation, cell growth and differentiation regulation (9-11). To the best of our knowledge, however, the effects of Huc-MSCs on the biological activities of endometrial glandular epithelial cells derived from patients with endometriosis have not previously been reported.

Epithelial-mesenchymal transition (EMT) refers to morphological and phenotypic transformation of epithelial cells to mesenchymal cells in response to stimulation of certain physiological or pathological changes, such as the loss of cell polarity, decreased contact with surrounding cells and the extracellular matrix, and increased migration and mobility (12). EMT is involved in early embryonic development and organogenesis, as well as wound healing (13). In addition, the metastasis of numerous types of malignant tumors, including lung cancer, is associated with EMT (14). EMT is a complex process by which epithelial cells acquire the characteristics of invasive mesenchymal cells (15).

The present study aimed to investigate whether exosomes derived from MSCs affect the migratory ability of endometrial glandular epithelial cells. In order to identify the underlying mechanism, the present study evaluated the effects of exosomes on EMT in endometrial glandular epithelial cells. 


\section{Materials and methods}

Tissue samples. The in situ endometrial tissue from five patients with endometriosis (age range, 31-42 years) and human umbilical cord tissue from six normal delivery woman (age range, 25-32 years) were provided by The Second Affiliated Hospital of Nanchang University (Nanchang, China) between June 2018 and July 2018. The experimental protocols were approved by the Ethics Committee of Nanchang University (China). All patients agreed to the use of their samples in scientific research and written informed consent was obtained from all patients.

Preparation of endometrial glandular epithelial cells. Ectopic endometrial tissue from patients with endometriosis was collected and cleaned using D-Hank's solution (cat. no. H1045; Beijing Solarbio Science \& Technology Co., Ltd.) containing antibiotics, and blood vessels and impurities in the tissue were shaved using a scalpel. Ophthalmic scissors were used to cut tissues into $1 \mathrm{~mm}^{3}$ blocks in a sterile Petri dish and the samples were transferred to a sterile centrifugal tube. Collagenase IV (0.1\%; Sigma-Aldrich; Merck KGaA) was added to the centrifugal tube and incubated at $37^{\circ} \mathrm{C}$ for 40-60 min. The digestion was terminated by adding DMEM with 20\% FBS (Hyclone; Cytiva) after intermittent oscillation. The solution was filtered through 100- and 400-mesh screens to remove large tissue fragments. The large tissue fragments on the surface of 100-mesh screen were transferred into a centrifugal tube, and collagenase $(0.1 \%)$ was added for a secondary digestion at $37^{\circ} \mathrm{C}$ for $5 \mathrm{~min}$. Cell suspension was collected, centrifuged $(1,000 \mathrm{x}$ g at room temperature for $10 \mathrm{~min}$ ), and suspended cells were inoculated on the culture plate. The cells were incubated at $37^{\circ} \mathrm{C}$ with $5 \% \mathrm{CO}_{2}$.

Preparation ofHuc-MSCs-derivedexosomes (Huc-MSCs-exo). The blood vessels in the Huc tissue were removed using a scalpel under a stereomicroscope, and tissues were washed with PBS, cut into $1 \mathrm{~mm}^{3}$ blocks and digested with diluted trypsin (PBS mixed with trypsin; $1: 1$ ) overnight at $4^{\circ} \mathrm{C}$. Tissue in the Petri dish was transferred to a $50 \mathrm{ml}$ Eppendorf tube and placed in a $37^{\circ} \mathrm{C}$ water bath for $15 \mathrm{~min}$. DMEM with $20 \%$ FBS was added to the Petri dish and cultured in an incubator for $15 \mathrm{~min}$ at $37^{\circ} \mathrm{C}$ and $5 \% \mathrm{CO}_{2}$. The cells were incubated with the following antibodies: Isothiocyanate (FITC) anti-CD34 (1:100; cat. no. 343604; BioLegend, Inc.), FITC anti-CD44 and FITC anti-CD45 (1:100; cat. nos. ab46793 and ab134199, respectively; both purchased from Abcam) at room temperature in the dark for $10 \mathrm{~min}$ and detected using a NovoCyte ${ }^{\mathrm{TM}}$ flow cytometer (NovoCyte 2060R; ACEA Bioscience, Inc; Agilent).

Extraction of Huc-MSCs-exo. Following $48 \mathrm{~h}$ starvation with FBS-free medium at $37^{\circ} \mathrm{C}$, exosome extraction kit was used to extract exosomes (cat. no. E1310; Bioruo) according to the manufacturer's instructions. Cells and debris were removed by centrifugation $\left(2,000 \mathrm{x} \mathrm{g} ; 4^{\circ} \mathrm{C} ; 10 \mathrm{~min}\right)$. The isolated exosomes were observed using transmission electron microscopy. The exosomes were fixed using $2.5 \%$ glutaraldehyde, phosphoric acid buffer preparation for $2 \mathrm{~h}$ at room temperature. After embedding, sections were cut (thickness, $70 \mathrm{~nm}$ ) and stained with 3\% uranium acetate and lead citrate for $10 \mathrm{~min}$ at room temperature. The slides were observed using transmission electron microscopy [JEM-1230 (80KV); JEOL, Ltd.], at magnification $x 1,000$. A total of $250 \mu \mathrm{g}$ Huc-MSCs-exo was labeled using PKH Lipophilic Membrane Dyes (cat. no. PKH67GL; Sigma-Aldrich, Merck KGaA) according to the manufacturer's instructions. PKH67-labeled Huc-MSCs-exo were centrifuged $\left(40,000 \mathrm{x} \mathrm{g} ; 4^{\circ} \mathrm{C} ; 70 \mathrm{~min}\right)$ and suspended in PBS $(50 \mu 1)$. Then, $2 \mu 1$ PKH67-labeled Huc-MSCs-exo solution was added to cells, which were incubated at $37^{\circ} \mathrm{C}$ for $0.5,2.0$ and $4.0 \mathrm{~h}$. The cells were imaged using Zeiss confocal laser scanning microscopy (LSM710; Zeiss GmbH), at magnification x200.

Experimental groups. The samples were divided into two groups ( $\mathrm{n}=6)$ : Control and Huc-MSCs-exo treatment $(10 \mu \mathrm{g} / \mathrm{ml})$ group. Subsequent experiments were performed $24 \mathrm{~h}$ after treatment.

Transwell assay. Following treatment with Huc-MSCs-exo for 24 and $48 \mathrm{~h}, 3 \times 10^{3}$ endometrial glandular epithelial cells were seeded in the upper chamber of Transwell plates (HyClone; GE Healthcare Life Sciences) with serum-free DMEM. The lower chamber contained DMEM with 10\% FBS (Hyclone; Cytiva). One day after seeding, the cells in the lower chamber were fixed with $4 \%$ paraformaldehyde for $30 \mathrm{~min}$ at room temperature and stained with $1 \%$ crystal violet (Beijing Solarbio Science \& Technology Co., Ltd.) for $5 \mathrm{~min}$ at room temperature. Images were captured using a light microscope (magnification, $\mathrm{x} 200$ ).

Wound healing assay. Endometrial glandular epithelial cells were seeded in 6-well plates ( $3 \times 10^{3}$ cells/well) in DMEM with $20 \%$ FBS, and treated with Huc-MSCs-exo for 24 and $48 \mathrm{~h}$. The cell monolayer was scratched. After 24 and $48 \mathrm{~h}$ incubation at $37^{\circ} \mathrm{C}$, images were captured using a light microscope (Olympus Corporation), at magnification x100.

Reverse transcription-quantitative (RT-q)PCR. At $24 \mathrm{~h}$ post-treatment, total RNA was extracted from endometrial glandular epithelial cells using an Ultrapure RNA extraction kit (CoWin Biosciences), according to the manufacturer's protocols. The purity of RNA was assessed by measuring optical density at 280/260 nmusing a spectrometer (LASPEC). RNA $(1 \mu \mathrm{g})$ was reverse transcribed into cDNA using an Avian Myeloblastosis Virus Reverse-Transcriptase kit (cat. no. KL041; Shanghai Kang Lang Biological Technology Co., Ltd.). The reaction system included $9.5 \mu 1$ RNase-free $\mathrm{dH}_{2} \mathrm{O}$, $1.0 \mu \mathrm{l} \mathrm{cDNA} / \mathrm{DNA}, 2.0 \mu \mathrm{l}$ primers and $12.5 \mu \mathrm{l}$ UltraSYBR mixture (cat. no. 00081405; CoWin Biosciences) and PCR was performed using the following thermocycling conditions: Initial denaturation at $95^{\circ} \mathrm{C} 10 \mathrm{~min}$; followed by 40 cycles of denaturation at $95^{\circ} \mathrm{C}$ for $10 \mathrm{sec}$, annealing at $58^{\circ} \mathrm{C}$ for $30 \mathrm{sec}$ and extension at $72^{\circ} \mathrm{C}$ for $30 \mathrm{sec}$; and final extension at $72^{\circ} \mathrm{C}$ for $3 \mathrm{~min}$. GAPDH was used as a reference gene. The following primers were used: E-cadherin forward, 5'-CTCACATTTCCCAACTCCTCT-3' and reverse, 5'-TGT CACCTTCAGCCATCCT-3'; Vimentin forward, 5'-GGA TTCACTCCCTCTGGTTG-3' and reverse, 5'-TGATGCTGA GAAGTTTCGTTG-3'; N-cadherin forward, 5'-GCTTAT 
A

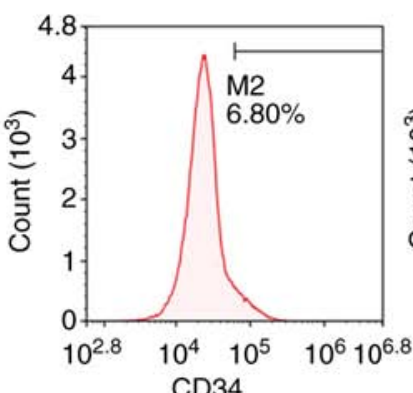

CD34

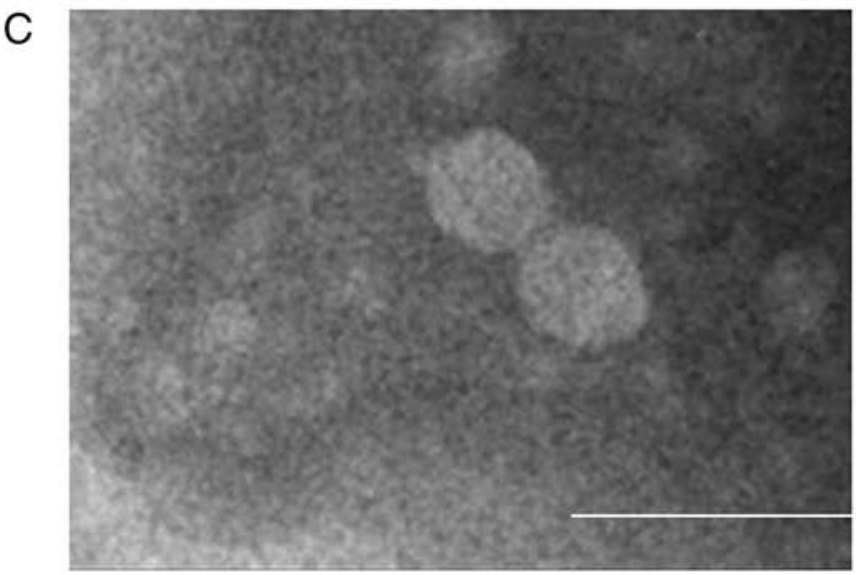

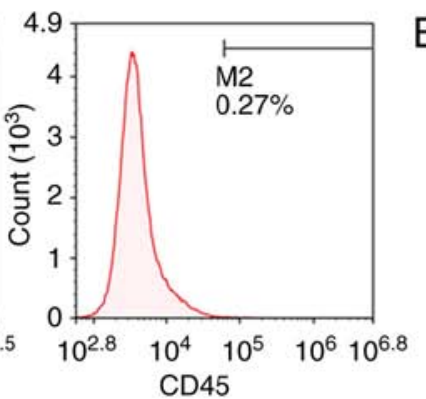

D

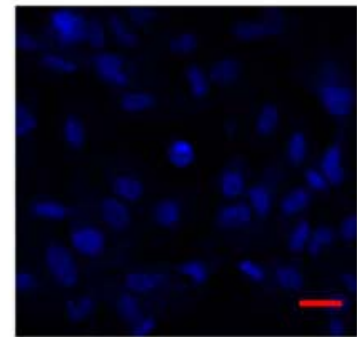

$0.5 \mathrm{~h}$

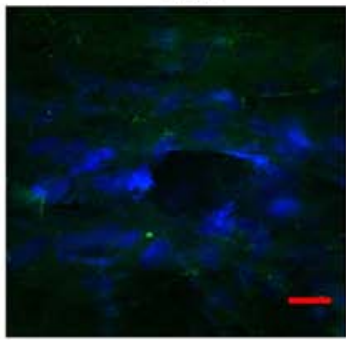

$2 \mathrm{~h}$
B
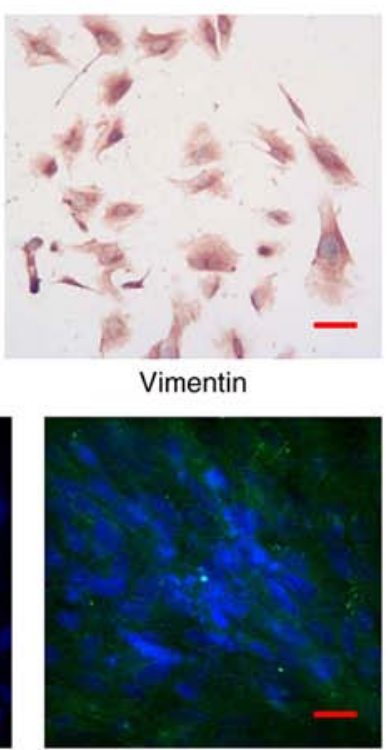

$1 \mathrm{~h}$

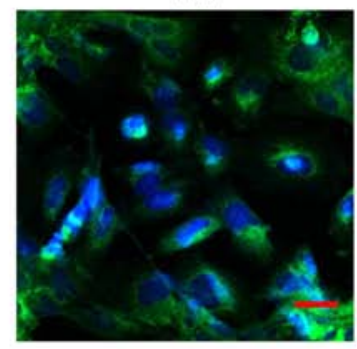

$4 \mathrm{~h}$

Figure 1. Labeling of Huc-MSCs-exo in endometrial glandular epithelial cells. (A) Flow cytometry for Huc-MSCs. (B) Immunohistochemistry for endometrial glandular epithelial cells. Scale bar, $100 \mu \mathrm{m}$. (C) Morphology of Huc-MSCs-exo detected using transmission electron microscopy. Scale bar, $50 \mathrm{~nm}$. (D) Labeling of HucMSCs-exo in endometrial glandular epithelial cells. Scale bar, $100 \mu \mathrm{m}$. Huc-MSCs-exo, human umbilical cord mesenchymal stem cell-derived exosomes.

CCTTGTGCTGATGTTT-3' and reverse, 5'-GTCTTCTTC TCCTCCACCTTCT-3'; and GAPDH forward, 5'-CAATGA CCCCTTCATTGACC-3' and reverse, 5'-GAGAAGCTT CCCGTTCTCAG-3'.

Western blotting. At $24 \mathrm{~h}$ post-treatment with Huc-MSCs-exo, protein was extracted from cells using the triplePrep kit (cat. no. 28-9425-44; ReadyPrep; GE Healthcare Life Sciences). The protein levels were quantified using a bicinchoninic acid protein assay kit (Beyotime Institute of Biotechnology), according to the manufacturer's instructions. A total of $25 \mu \mathrm{g} /$ lane protein was separated via SDS-PAGE on a $10 \%$ gel and transferred onto nitrocellulose membranes. The membranes were blocked using 5\% skimmed milk at room temperature for $2 \mathrm{~h}$ and incubated overnight at $4^{\circ} \mathrm{C}$ with the following primary antibodies: Rabbit polyclonal anti-E-cadherin (1:1,500; cat. no. AF0131; Affinity Biosciences), anti-GAPDH (1:1,000; cat. no. AG019; Beyotime Institute of Biotechnology), anti-Vimentin and anti-N-cadherin (1:3,000 and 1:1,000, respectively; cat. nos. ab92547 and ab76057, respectively; both Abcam). After washing with $0.1 \mathrm{M}$ PBS, the membranes were incubated with the secondary antibody (HRP-labeled goat anti-rabbit IgG; cat. no. A16104; Thermo Fisher Scientific, Inc.) at $4^{\circ} \mathrm{C}$ for $2 \mathrm{~h}$. The blots were visualized using an electrochemiluminescence kit (Thermo Fisher Scientific, Inc.). The densities of the blots were quantified using the Quantity One software (version 4.62; Bio-Rad Laboratories, Inc.).

Immunohistochemistry. The cells were fixed in paraformaldehyde for $30 \mathrm{~min}$ at room temperature. Staining was performed using monoclonal antibodies against $\mathrm{N}$-cadherin, Vimentin (both 1:100; cat nos. ab76057 and ab92547, respectively; both Abcam) and E-cadherin (1:200; cat. no. AF0131; Affinity Biosciences). Endogenous peroxidase activity was blocked with $3 \%(\mathrm{v} / \mathrm{v}) \mathrm{H}_{2} \mathrm{O}_{2}$ for $5 \mathrm{~min}$ at room temperature. The slides were incubated with the aforementioned primary antibodies overnight at $4^{\circ} \mathrm{C}$, followed by incubation with horseradish peroxidase-labeled goat anti-rabbit IgG secondary antibody (1:10,000; cat. no. A16104SAMPLE; Thermo Fisher Scientific, Inc.) for $30 \mathrm{~min}$ at room temperature. Cells were stained with 3,3'-diaminobenzidine chromogen for $3 \mathrm{~min}$ at room temperature. Images were captured using a light microscope (BX51; Olympus Corporation), at magnification, x200.

Statistical analysis. Data are expressed as the mean \pm standard deviation of six independent experiments. All statistical analysis was performed using unpaired Student's t-test using GraphPad Prism (version 7.0; GraphPad Software, Inc.). $\mathrm{P}<0.05$ was considered to indicate a statistically significant difference. 

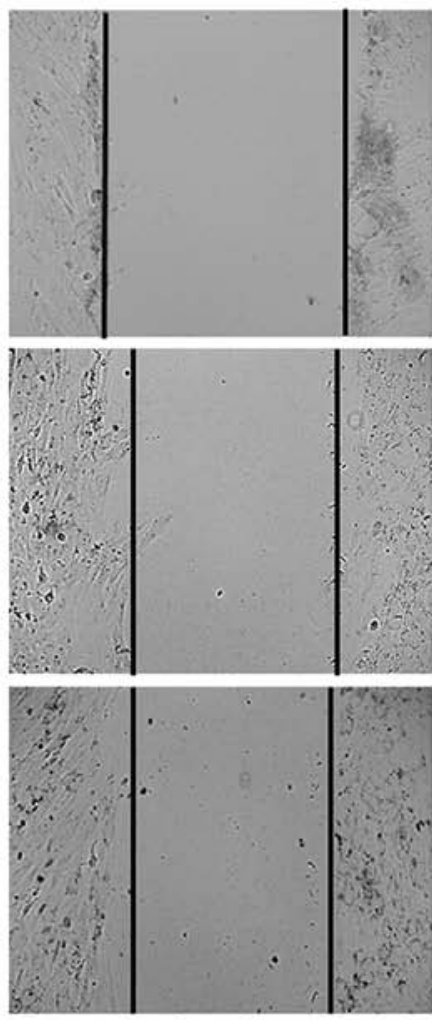

Control
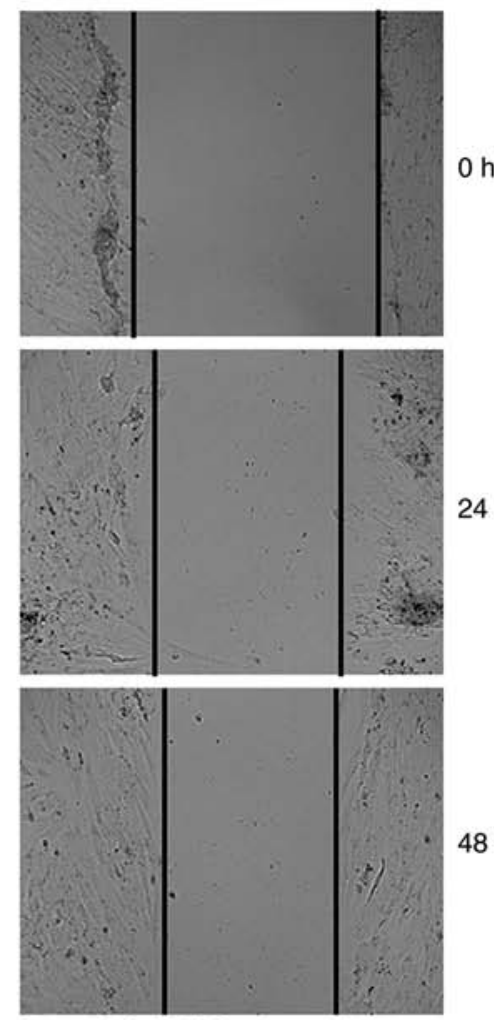

hucMSCs-exo

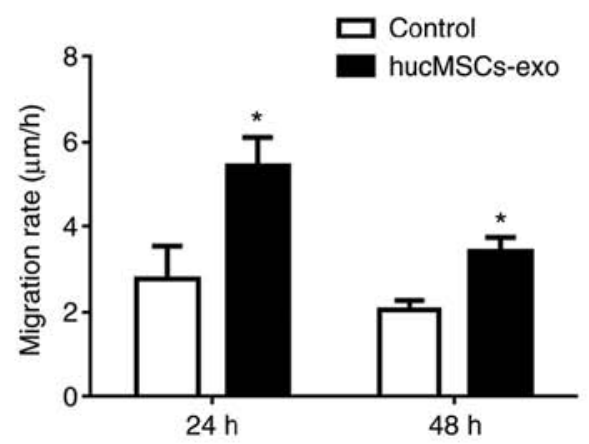

Figure 2. Huc-MSCs-exo facilitate wound healing ability of endometrial glandular epithelial cells. Left, representative images of cell migration (magnification, $\mathrm{x} 100$ ); right, quantification data. $\mathrm{P}<0.05$ vs. control. Huc-MSCs-exo, human umbilical cord mesenchymal stem cell-derived exosomes.

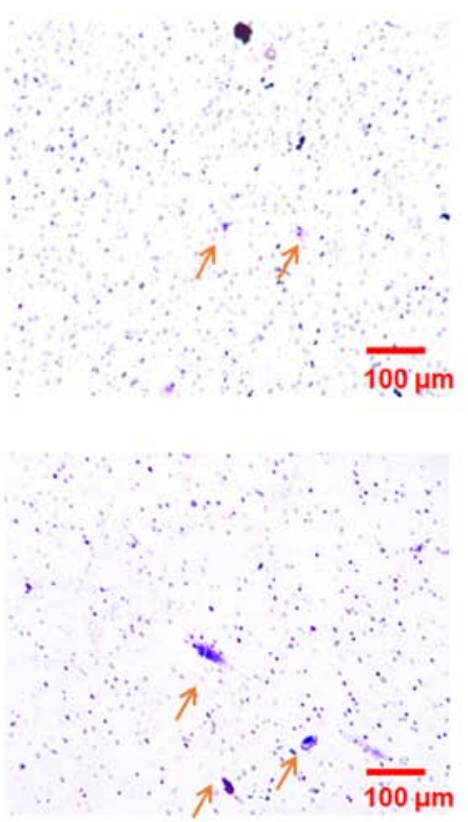

Control

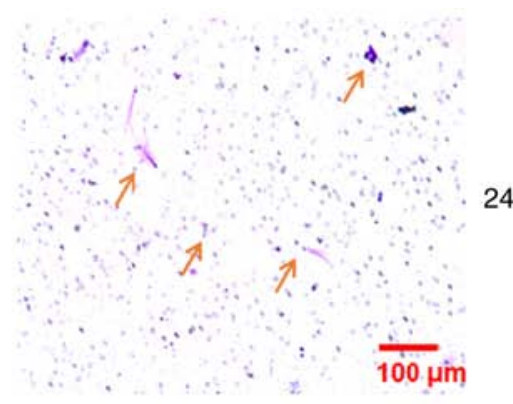

$24 \mathrm{~h}$
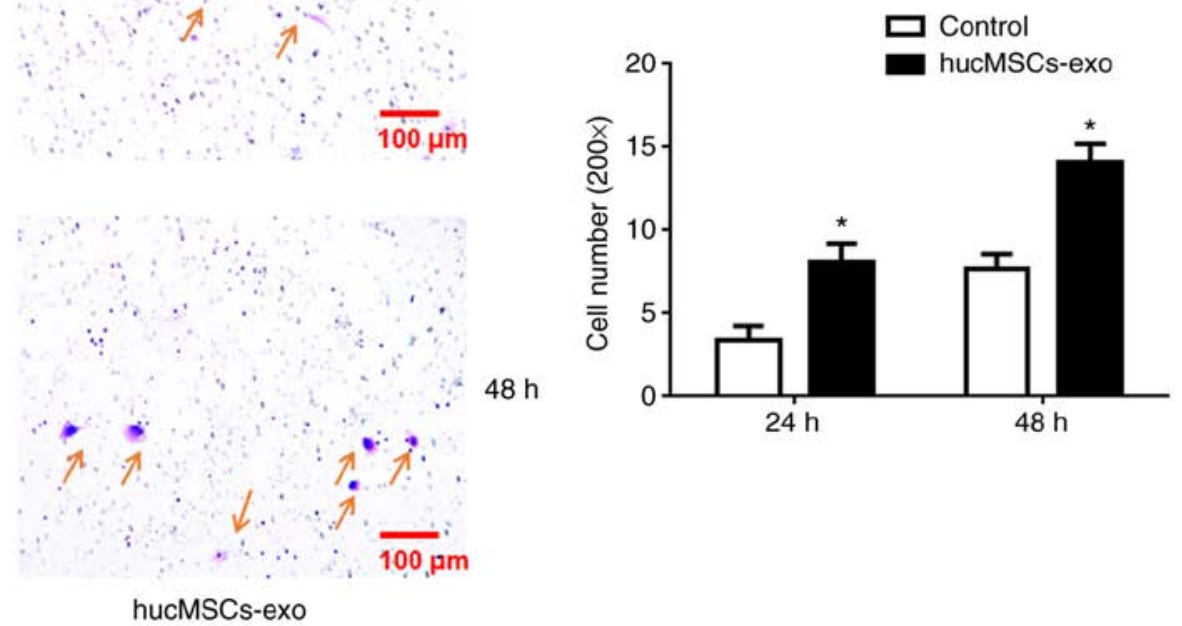

Figure 3. Huc-MSCs-exo facilitate migratory ability of endometrial glandular epithelial cells. Arrows indicate migratory cells. ${ }^{*} \mathrm{P}<0.05$ vs. control. Huc-MSCs-exo, human umbilical cord mesenchymal stem cell-derived exosomes.

\section{Results}

Identification of Huc-MSCs and endometrial glandular epithelial cells. CD44, CD34 and CD45 expression levels in the isolated cells were $92.40,6.80$ and $0.27 \%$, respectively
(Fig. 1A). These data indicated that the isolated cells were $\mathrm{CD}_{4}{ }^{+}, \mathrm{CD} 34^{-}$and $\mathrm{CD} 45^{-}$, which revealed the successful isolation of umbilical cord-derived MSCs. Cells expressed vimentin (dark brown), which indicated that endometrial glandular epithelial cells were successfully isolated (Fig. 1B). 
A
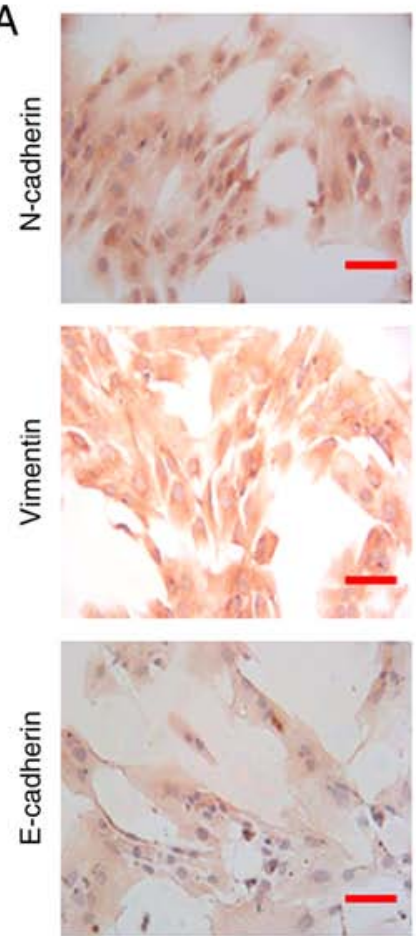

Control
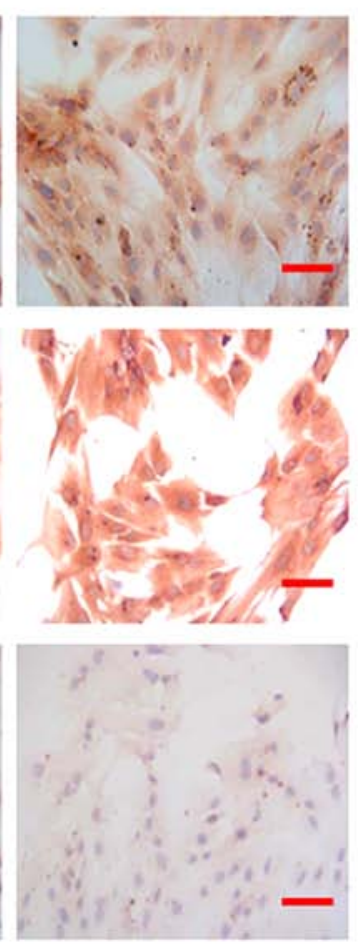

hucMSCs-exo

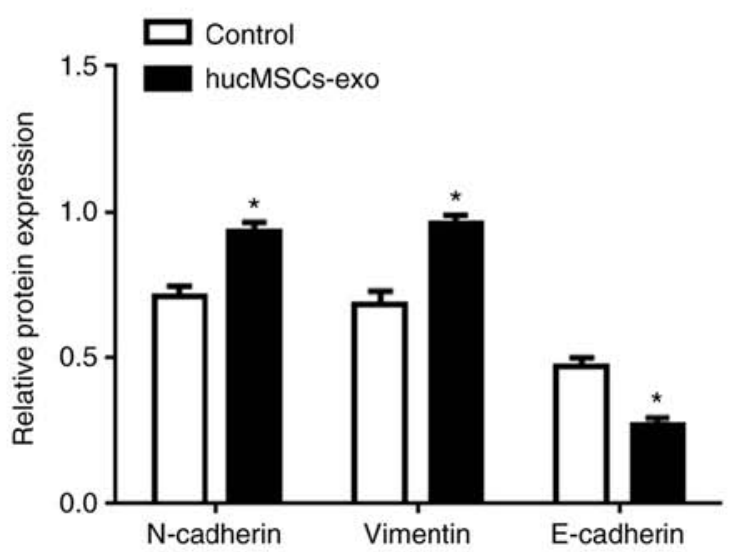

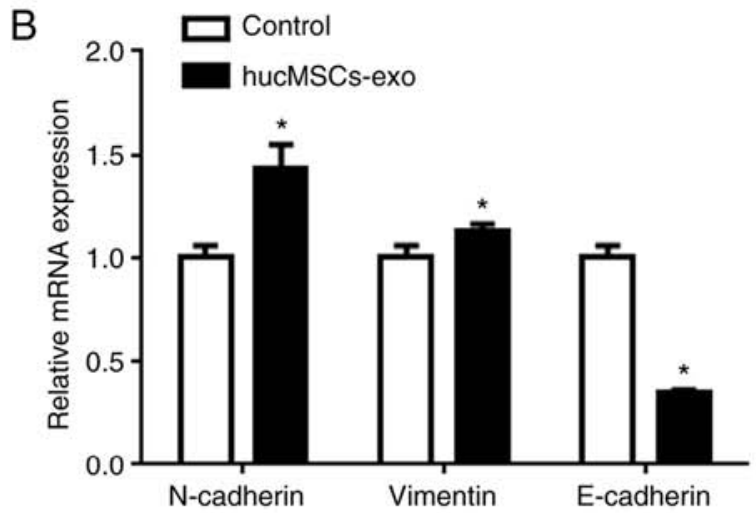
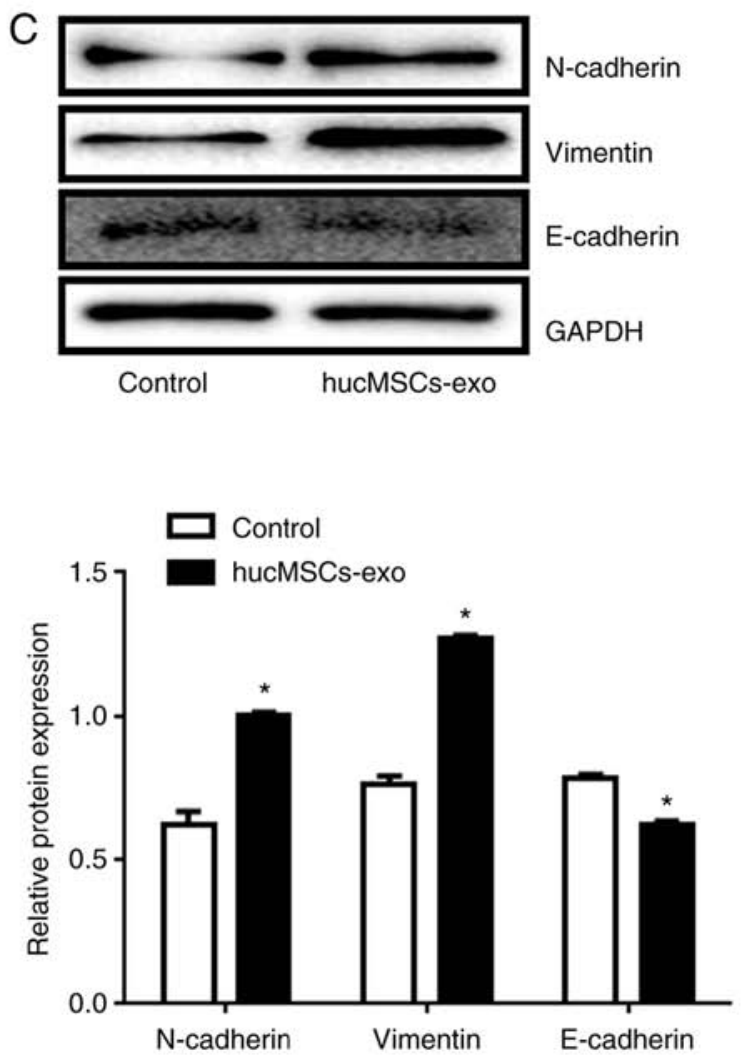

Figure 4. Huc-MSCs-exo increase expression levels of N-cadherin and Vimentin, and decrease E-cadherin expression levels. (A) N-cadherin, Vimentin and E-cadherin expression levels detected by immunohistochemistry. Scale bar, $100 \mu \mathrm{m}$. (B) mRNA expression levels detected via reverse transcriptionquantitative PCR. (C) Protein expression levels detected via western blotting. "P $<0.05$ vs. control. Huc-MSCs-exo, human umbilical cord mesenchymal stem cell-derived exosomes.

Labeling of Huc-MSCs-exo in endometrial glandular epithelial cells via transmission electron microscopy. Exosomes exhibited typical round or oval cup-shaped structures with complete morphology (Fig. 1C). Huc-MSCs-exo entered endometrial glandular epithelial cells over time (Fig. 1D). At $4 \mathrm{~h}$, the labeled exosomes were notably expressed in the cytoplasm.

Huc-MSCs-exo facilitate the wound healing ability of endometrial glandular epithelial cells. As shown in Fig. 2, the migratory ability of endometrial glandular epithelial cells treated with Huc-MSCs-exo was significantly enhanced at 24 and $48 \mathrm{~h}$ compared with that of the control group $(\mathrm{P}<0.05)$, which demonstrated that Huc-MSCs exosomes promote the migration of endometrial glandular epithelial cells.
Huc-MSCs-exo facilitate the migratory ability of endometrial glandular epithelial cells. Compared with control group, the migratory ability of HucMSCs-exo-treated cells significantly increased at 24 and $48 \mathrm{~h}(\mathrm{P}<0.05$; Fig. 3), which demonstrated that Huc-MSCs-exo promote the migratory ability of endometrial glandular epithelial cells.

Huc-MSCs-exo increase expression levels of $\mathrm{N}$-cadherin and Vimentin, and decrease E-cadherin expression levels. Immunohistochemical staining demonstrated that the relative expression levels of $\mathrm{N}$-cadherin and Vimentin increased and the expression level of E-cadherin decreased in the Huc-MSCs-exo group compared with the control group $(\mathrm{P}<0.05$; Fig. 4A). RT-qPCR also demonstrated that Huc-MSCs-exo treatment promoted expression levels of $\mathrm{N}$-cadherin and Vimentin and 
decreased expression levels of E-cadherin at the mRNA level (Fig. 4B). This was consistent with the results of the western blotting analysis (Fig. 4C).

\section{Discussion}

The present study demonstrated that Huc-MSCs-exo promoted the migration of endometrial glandular epithelial cells from patients with endometriosis at both 24 and $48 \mathrm{~h}$. It was also demonstrated that treatment with Huc-MSCs-exo inhibited expression levels of E-cadherin and promoted expression levels of Vimentin and $\mathrm{N}$-cadherin. The results of the present study indicate the potential function of Huc-MSCs-exo in patients with endometriosis.

Endometriosis is a common gynecological disease, primarily occurring in women of child-bearing age (25-45 years). It is estimated that 10.8 million individuals were affected globally as of 2015 (16). The primary clinical symptoms include dysmenorrhea, increased menstrual volume and infertility, which may adversely affect the quality of life of patients (17). In the present study, endometrial glandular epithelial cells were isolated and identified via immunohistochemistry. MSCs are adult stem cells that originate from the embryonic mesoderm and possess strong self-renewal ability and multi-directional differentiation potential (18). Numerous studies have demonstrated that MSCs can serve as the host of primary or metastatic tumors and promote the formation of tumor microenvironment $(19,20)$. Studies have demonstrated that MSCs can either promote or inhibit the growth of tumors $(21,22)$. MSCs may promote the development of cancer via EMT, which is characterized by the transformation of cells from epithelial to mesenchymal phenotype (23). In the present study, Huc-MSCs were isolated from Huc tissues and identified via flow cytometry using specific antibodies as previously described (23).

Exosomes from numerous cell sources contain biologically active components, such as cell membrane molecules and cytoplasmic proteins (24). Intercellular information is transferred via fusion with the cell membranes of adjacent cells (25). Herrera et al (26) demonstrated that exosomes derived from human stem cells accelerate liver regeneration in hepatectomized rats. Exosomes are considered to be potential mediators for inducing peripheral tolerance and regulating the immune response (27). Exosomes are considered to be mediators of intercellular communication, and influence target receptor cells by inducing intracellular signal transduction or endowing donor cells with novel substances, such as protein or mRNA (9-11). In the present study, exosomes were identified using transmission electron microscopy. It was demonstrated that the exosomes were round or elliptical in shape and conformed to typical morphological characteristics. The labeling of endometrial glandular epithelial cells by exosomes demonstrated that the majority of the exosomes entered cells, allowing them to exert their function.

Endometriosis is a non-cancerous disease with invasive ability. The invasiveness of endometrial cells serves a key role in the occurrence and development of endometriosis. Huc-MSCs-exo promote the migratory and invasive abilities of A549 cells (23). Numerous studies have demonstrated that MSC-exo exerts both anti-tumor and pro-tumor effects in human breast, ovarian, gastric and nasopharyngeal cancer, as well as in multiple myeloma, osteosarcoma and rat liver cancer (28-36). MSC-exo also promotes migration and invasion of human lung cancer cells (23). The results of the present study demonstrated that Huc-MSCs-exo promote the migration of endometrial glandular epithelial cells.

EMT refers to the loss of epithelial characteristics and acquisition of mesenchymal phenotypes. When cells undergo EMT, they detach from other cells, decrease expression levels of epithelial markers and acquire mesenchymal characteristics, such as enhanced mobility and invasiveness (37,38). Zhu et al demonstrated that human MSC-conditioned medium induces EMT in cancer cells $(39,40)$. The active component of conditioned medium is exosome from mesenchymal stem cells $(39,40)$. A previous study demonstrated that expression levels of EMT markers are altered by exosomes in vivo (36). Bartley et al (41) demonstrated that expression levels of E-cadherin are absent in certain glandular epithelial cells in ectopic lesions of patients with endometriosis, which indicates that EMT may also play a key role in the formation and progression of endometriosis. EMT-associated transcription factors can directly or indirectly inhibit expression levels of E-cadherin and promote expression levels of N-cadherin and Vimentin in mesenchymal phenotypes, thereby promoting EMT (42). The results of the present study demonstrated that Huc-MSCs-exo promote EMT and upregulate expression levels of $\mathrm{N}$-cadherin and Vimentin, while inhibiting expression levels of E-cadherin. This is consistent with the aforementioned results and supports the hypothesis that Huc-MSCs-exo alter expression levels of EMT-associated genes, thus enhancing the migratory and invasive abilities of endometrial glandular epithelial cells.

In the present study, Huc-MSCs-exo promoted the migratory ability of endometrial glandular epithelial cells from patients with endometriosis. The current results indicate the potential application of Huc-MSCs-exo in the treatment of endometriosis. Further research is required to quantify the dose effect of Huc-MSCs-exo and to investigate the underlying mechanism. Huc-MSCs-exo function should also be further evaluated in an in vivo model.

In conclusion, Huc-MSCs-exo promote the migratory ability of endometrial glandular epithelial cells, potentially via promoting EMT in endometrial glandular epithelial cells.

\section{Acknowledgements}

Not applicable.

\section{Funding}

Not applicable.

\section{Availability of data and materials}

The datasets used and/or analyzed during the current study are available from the corresponding author on reasonable request.

\section{Authors' contributions}

YF, FZ and YZ performed the experiments and analyzed the data. YF and BT designed the study and wrote the manuscript. All authors read and approved the final manuscript. 


\section{Ethics approval and consent to participate}

The experimental protocols were approved by the Ethics Committee of Nanchang University (approval no. 2018071401).

\section{Patient consent for publication}

All patients agreed to the use of their samples in scientific research and written informed consent was obtained from all patients.

\section{Competing interests}

The authors declare that they have no competing interests.

\section{References}

1. Vercellini P, Viganò P, Somigliana E and Fedele L: Endometriosis: Pathogenesis and treatment. Nat Rev Endocrinol 10: 261-275, 2014.

2. Mehedintu C, Plotogea MN, Ionescu S and Antonovici M: Endometriosis still a challenge. J Med Life 7: 349-357, 2014.

3. Peiris AN, Chaljub E and Medlock D: Endometriosis. JAMA 320 : 2608, 2018

4. Berkley KJ, Rapkin AJ and Papka RE: The pains of endometriosis. Science 308: 1587-1589, 2005.

5. Matsuzaki S and Darcha C: Co-operation between the AKT and ERK signaling pathways may support growth of deep endometriosis in a fibrotic microenvironment in vitro. Hum Reprod 30: $1606-1616,2015$

6. Orbay H, Tobita M and Mizuno H: Mesenchymal stem cells isolated from adipose and other tissues: Basic biological properties and clinical applications. Stem Cells Int 2012: 461718, 2012.

7. Li X, Wang Q, Ding L, Wang YX, Zhao ZD, Mao N, Wu CT, Wang H, Zhu H and Ning SB: Intercellular adhesion molecule-1 enhances the therapeutic effects of MSCs in a dextran sulfate sodium-induced colitis models by promoting MSCs homing to murine colons and spleens. Stem Cell Res Ther 10: 267, 2019.

8. Samanta S, Rajasingh S, Drosos N, Zhou Z, Dawn B and Rajasingh J: Exosomes: New molecular targets of diseases. Acta Pharmacol Sin 39: 501-513, 2018.

9. Li T, Yan Y, Wang B, Qian H, Zhang X, Shen L, Wang M, Zhou Y, Zhu W, Li W and Xu W: Exosomes derived from human umbilical cord mesenchymal stem cells alleviate liver fibrosis. Stem Cells Dev 22: 845-854, 2013.

10. Jiang L, Zhang S, Hu H, Yang J, Wang X, Ma Y, Jiang J, Wang J, Zhong L, Chen M, et al: Exosomes derived from human umbilical cord mesenchymal stem cells alleviate acute liver failure by reducing the activity of the NLRP3 inflammasome in macrophages. Biochem Biophys Res Commun 508: 735-741, 2019.

11. Zhang Y, Hao Z, Wang P, Xia Y, Wu J, Xia D, Fang S and Xu S: Exosomes from human umbilical cord mesenchymal stem cells enhance fracture healing through HIF-1 $\alpha$-mediated promotion of angiogenesis in a rat model of stabilized fracture. Cell Prolif 52: e12570, 2019.

12. Pastushenko I, Brisebarre A, Sifrim A, Fioramonti M, Revenco T, Boumahdi S, Van Keymeulen A, Brown D, Moers V, Lemaire $\mathrm{S}$, et al: Identification of the tumour transition states occurring during EMT. Nature 556: 463-468, 2018.

13. Barriere G, Fici P, Gallerani G, Fabbri F and Rigaud M: Epithelial mesenchymal transition: A double-edged sword. Clin Transl Med 4: 14, 2015.

14. Liang H, Yu T, Han Y, Jiang H, Wang C, You T, Zhao X, Shan H, Yang R, Yang L, et al: LncRNA PTAR promotes EMT and invasion-metastasis in serous ovarian cancer by competitively binding miR-101-3p to regulate ZEB1 expression. Mol Cancer 17: $119,2018$.

15. Son $\mathrm{H}$ and Moon A: Epithelial-mesenchymal transition and cell invasion. Toxicol Res 26: 245-252, 2010.

16. GBD 2015 Disease and Injury Incidence and Prevalence Collaborators: Global, regional, and national incidence, prevalence, and years lived with disability for 310 diseases and injuries, 1990-2015: A systematic analysis for the global burden of disease study 2015. Lancet 388: 1545-1602, 2016
17. Mboi N, Murty Surbakti I, Trihandini I, Elyazar I, Houston Smith K, Bahjuri Ali P, Kosen S, Flemons K, Ray SE, Cao J, et al: On the road to universal health care in Indonesia, 1990-2016: A systematic analysis for the global burden of disease study 2016 . Lancet 392: 581-591, 2018.

18. Marrazzo P, Crupi AN, Alviano F, Teodori L and Bonsi L: Exploring the roles of MSCs in infections: Focus on bacterial diseases. J Mol Med (Berl) 97: 437-450, 2019.

19. Droujinine IA, Eckert MA and Zhao W: To grab the stroma by the horns: From biology to cancer therapy with mesenchymal stem cells. Oncotarget 4: 651-664, 2013.

20. Kidd S, Spaeth E, Dembinski JL, Dietrich M, Watson K, Klopp A, Battula VL, Weil M, Andreeff M and Marini FC: Direct evidence of mesenchymal stem cell tropism for tumor and wounding microenvironments using in vivo bioluminescent imaging. Stem Cells 27: 2614-2623, 2009.

21. Klopp AH, Gupta A, Spaeth E, Andreeff M and Marini F III: Concise review: Dissecting a discrepancy in the literature: do mesenchymal stem cells support or suppress tumor growth? Stem Cells 29: 11-19, 2011

22. Rhee KJ, Lee JI and Eom YW: Mesenchymal stem cell-mediated effects of tumor support or suppression. Int J Mol Sci 16: 30015-30033, 2015.

23. Zhao X, Wu X, Qian M, Song Y, Wu D and Zhang W: Knockdown of TGF- $\beta 1$ expression in human umbilical cord mesenchymal stem cells reverts their exosome-mediated EMT promoting effect on lung cancer cells. Cancer Lett 428: 34-44, 2018.

24. Cocucci E, Racchetti G and Meldolesi J: Shedding microvesicles: Artefacts no more. Trends Cell Biol 19: 43-51, 2009.

25. Schneider A and Simons M: Exosomes: Vesicular carriers for intercellular communication in neurodegenerative disorders. Cell Tissue Res 352: 33-47, 2013.

26. Herrera MB, Fonsato V, Gatti S, Deregibus MC, Sordi A Cantarella D, Calogero R, Bussolati B, Tetta C and Camussi G: Human liver stem cell-derived microvesicles accelerate hepatic regeneration in hepatectomized rats. J Cell Mol Med 14: 1605-1618, 2010.

27. Mokarizadeh A, Delirezh N, Morshedi A, Mosayebi G, Farshid AA and Mardani K: Microvesicles derived from mesenchymal stem cells: Potent organelles for induction of tolerogenic signaling. Immunol Lett 147: 47-54, 2012.

28. Pakravan K, Babashah S, Sadeghizadeh M, Mowla SJ, Mossahebi-Mohammadi M, Ataei F, Dana N and Javan M: MicroRNA-100 shuttled by mesenchymal stem cell-derived exosomes suppresses in vitro angiogenesis through modulating the mTOR/HIF-1 $\alpha /$ VEGF signaling axis in breast cancer cells. Cell Oncol (Dordr) 40: 457-470, 2017.

29. Ono M, Kosaka N, Tominaga N, Yoshioka Y, Takeshita F, Takahashi RU, Yoshida M, Tsuda H, Tamura K and Ochiya T: Exosomes from bone marrow mesenchymal stem cells contain a microRNA that promotes dormancy in metastatic breast cancer cells. Sci Signal 7: ra63, 2014.

30. Reza AMMT, Choi YJ, Yasuda H and Kim JH: Human adipose mesenchymal stem cell-derived exosomal-miRNAs are critical factors for inducing anti-proliferation signalling to A2780 and SKOV-3 ovarian cancer cells. Sci Rep 6: 38498, 2016.

31. Ko SF, Yip HK, Zhen YY, Lee CC, Lee CC, Huang CC, Ng SH and Lin JW: Adipose-derived mesenchymal stem cell exosomes suppress hepatocellular carcinoma growth in a rat model: Apparent diffusion coefficient, natural killer T-cell responses, and histopathological features. Stem Cells Int 2015: 853506, 2015.

32. Wang J, Hendrix A, Hernot S, Lemaire M, De Bruyne E, Van Valckenborgh E, Lahoutte T, De Wever O, Vanderkerken K and Menu E: Bone marrow stromal cell-derived exosomes as communicators in drug resistance in multiple myeloma cells. Blood 124: 555-566, 2014.

33. Gu H, Ji R, Zhang X, Wang M, Zhu W, Qian H, Chen Y, Jiang P and $\mathrm{Xu}$ W: Exosomes derived from human mesenchymal stem cells promote gastric cancer cell growth and migration via the activation of the Akt pathway. Mol Med Rep 14: 3452-3458, 2016.

34. Ji R, Zhang B, Zhang X, Xue J, Yuan X, Yan Y, Wang M, Zhu W, Qian $\mathrm{H}$ and $\mathrm{Xu} \mathrm{W}$ : Exosomes derived from human mesenchymal stem cells confer drug resistance in gastric cancer. Cell Cycle 14: 2473-2483, 2015

35. Qi J, Zhou Y, Jiao Z, Wang X, Zhao Y, Li Y, Chen H, Yang L, $\mathrm{Zhu} \mathrm{H}$ and $\mathrm{Li} \mathrm{Y}$ : Exosomes derived from human bone marrow mesenchymal stem cells promote tumor growth through hedgehog signaling pathway. Cell Physiol Biochem 42: 2242-2254, 2017 
36. Shi S, Zhang Q, Xia Y, You B, Shan Y, Bao L, Li L, You Y and Gu Z Mesenchymal stem cell-derived exosomes facilitate nasopharyngeal carcinoma progression. Am J Cancer Res 6: 459-472, 2016.

37. Polyak K and Weinberg RA: Transitions between epithelial and mesenchymal states: Acquisition of malignant and stem cell traits. Nat Rev Cancer 9: 265-273, 2009.

38. Acloque H, Adams MS, Fishwick K, Bronner-Fraser M and Nieto MA: Epithelial-mesenchymal transitions: The importance of changing cell state in development and disease. J Clin Invest 119: 1438-1449, 2009.

39. Zhu W, Huang L, Li Y, Qian H, Shan X, Yan Y, Mao F, Wu X and Xu WR: Mesenchymal stem cell-secreted soluble signaling molecules potentiate tumor growth. Cell Cycle 10: 3198-3207, 2011

40. Zhu W, Huang L, Li Y, Zhang X, Gu J, Yan Y, Xu X, Wang M, Qian $\mathrm{H}$ and $\mathrm{Xu} \mathrm{W}$ : Exosomes derived from human bone marrow mesenchymal stem cells promote tumor growth in vivo. Cancer Lett 315: 28-37, 2012.
41. Bartley J, Jülicher A, Hotz B, Mechsner S and Hotz H: Epithelial to mesenchymal transition (EMT) seems to be regulated differently in endometriosis and the endometrium. Arch Gynecol Obstet 289: 871-881, 2014.

42. Loh CY, Chai JY, Tang TF, Wong WF, Sethi G, Shanmugam MK, Chong PP and Looi CY: The E-cadherin and N-cadherin switch in epithelial-to-mesenchymal transition: Signaling, therapeutic implications, and challenges. Cells 8: pii: E1118, 2019.

(i) (5) This work is licensed under a Creative Commons Attribution-NonCommercial-NoDerivatives 4.0 International (CC BY-NC-ND 4.0) License. 